\title{
Conference Organisation
}

\section{ISfT Executive Committee}

- Steinar Pedersen, Norway, President ISfT

- Ace Allen, USA

- André Lacroix, Canada

- Guy Harris, Japan

- Louis Lareng, France

- Patrick Barron, Japan

- Takashi Takahashi, Japan

\section{Scientific Committee}

- Hans Kollberg, Sweden, Chairman and Conference President

- Toomas Asser, Estonia

- Rashid Bashshur, USA

- Björn Bergh, Germany

- Knut Bernstein, Denmark

- Egil Bovim, KoKom, Norway

- Peter Buxton, United Kingdom

- Stefan Cajander, Sweden

- Li-Qun Cheng, P.R of China

- Russel D'Souza, Australia

- Kenneth Eaton, United Kingdom

- Rod Elford, Canada

- Uwe Engelmann, Germany

- Magnus Hjelm, United Kingdom

- Lars Hulbäck, Denmark

- Olof Jarlman, Sweden

- Zviad Kirtava, Georgia

- K.C Lun, Singapore

- Salah H. Mandil, Switzerland

- James McGee, United Kingdom

- Leif Eric Nohr, Norway

- Jaime A. Cortes Ojeda, Costa Rica

- Yoshikazu Okada, Japan

\section{Local Organising Committee}

- Björn-Erik Erlandson, Chairman

- Hans Kollberg, Conference President

- Tia Eriksson, Conference Manager

- Hans Adolfsson

- Göran Brodin

- Stefan Cajander

- Anders Fallgren

- Sabine Koch
- Oleg Orlov, Russia

- Dittmar Padeken, Germany

- Thorgeir Palsson, Iceland

- Eduardo Perez- Bercoff, Sweden

- Ron Poropatich, USA

- Jarmo Reponen, Finland

- Ricky J. Richardson, United Kingdom

- Rick Satava, USA

- Monique Savoldelli, France

- Werner Schneider, Sweden

- Sanjay P. Sood, India

- Benedict Stanberry, United Kingdom

- Thomas Stensgaard, Greenland

- Erik Stålberg, Sweden

- Geert Thienpont, Belgium

- Thorkild Tylleskär, Sweden

- Siri Uldal, Norway

- Arnolds Veinbergs, Latvia

- Ole Winding, Denmark

- Richard Wooton, United Kingdom

- Peter Yellowlees, Australia 


\section{Sponsors}

- AVP Moebius

- Tieto Enator

- Proffice Care

- Agfa 